\title{
LA VALORACION DE LOS EXPERTOS SOBRE LAS ESTADISTICAS RELATIVAS AL CONOCIMIENTO DE LOS TURISTAS ${ }^{1}$
}

\author{
María Pilar Peñarrubia-Zaragoza* \\ Universidad de Valencia \\ https://orcid.org/0000-0001-8478-8314
}

\section{RESUMEN}

El conocimiento del comportamiento del turista, a partir de la información que generan y recogen tanto administraciones públicas, como privadas, resulta clave para la planificación de los destinos. Por ello, es fundamental conocer la opinión de los expertos al respecto. La realización de entrevistas personales a diferentes actores de la administración pública, la empresa privada y el ámbito universitario ha permitido detectar necesidades y demandas reales de información en relación al comportamiento del turista, así como realizar una reflexión relativa a la necesidad de la formulación de un Sistema de Información Turístico público.

Palabras clave: comportamiento del turista; fuentes estadísticas públicas; fuentes de datos tecnológicos; inteligencia turística; expertos turísticos.

\section{How esperts assess statistics regarding knowledge about tourist}

\section{ABSTRACT}

Knowledge of tourist behavior, based on the information generated and collected by both public and private management, is key for destination planning. Therefore, in this regard, it is essential to know the experts' opinions. Carrying out personal interviews with different players in the public administration, private companies and the academy has enabled finding

Fecha de recepción: 31 de marzo de 2020.

Fecha de aceptación: 25 de mayo de 2020.

* Departamento de Geografía. Universidad de Valencia. Avda. Blasco Ibáñez, 28. 46010 VALENCIA (España).E-mail: m.pilar.penarrubia@uv.es

$1 \quad{ }^{1}$ La investigación que da lugar a este trabajo constituye uno de los resultados del proyecto "Análisis de la sostenibilidad Urbana como estrategia de regeneración del espacio público de las áreas turísticas del litoral", financiado por la Fundación CajaCanarias, cuyo investigador principal es Moisés Simancas Cruz, Profesor Titular de Geografía Humana la Universidad de La Laguna. 
actual needs and requirements of information regarding tourist behaviors, as well as reflecting on the necessity of the development of a public Tourist Information System.

Keywords: tourist behavior; public statistical sources; technological data sources; tourist intelligence; tourism experts.

\section{LA IMPORTANCIA DE LOS DATOS TURÍSTICOS EN LA TOMA DE DECI- SIONES}

El conocimiento del comportamiento del consumidor resulta fundamental tanto para empresas, como instituciones públicas ayudando al diseño y orientación de estrategias de planificación y políticas territoriales. Éste se define como "las actividades que las personas efectúan al obtener, consumir y disponer de productos y servicios", entendiendo como obtención, las actividades que llevan a la compra o recepción de un producto; consumir a cómo, dónde, cuándo y bajo qué circunstancias los consumidores utilizan los productos; y disponer incluye la forma en que los consumidores se deshacen de productos y empaques (Blackwell, Miniard y Engel, 2002).

El comportamiento del turista en la compra de productos y servicios turísticos presenta características específicas que justifican la singularidad y complejidad del proceso. Serra (2002) identifica de forma esquemática seis características principales: a) el elevado componente emocional, considerado por muchas personas como un factor relevante a la hora de valorar su "calidad de vida"; b) el elevado nivel de implicación y compromiso en la compra; c) el elevado nivel de incertidumbre derivado de la intangibilidad, en cuanto que se trata de productos y servicios que no pueden ser probados antes de la compra; d) la fuerte influencia de otras personas; e) la antelación en la decisión de compra; y f) el elevado nivel de búsqueda de información. La patente variedad de factores evidencia la complejidad del proceso de conocimiento del cliente y hace necesaria su investigación con el objetivo de planificar el sector orientado al cliente.

A estas características podemos añadir el planteamiento que "el consumo turístico es visto por muchos segmentos de mercado, por muchos consumidores, como la acumulación continua de experiencias, lo cual se consigue con la variedad de destinos y evitando la repetición, pese a no ser compras de baja implicación" (Escobar y González, 2011: 253). Esta afirmación justifica la necesidad de proceder a la renovación cíclica de las actividades ofrecidas en los destinos turísticos, con el objetivo de que los turistas que repitan tengan la sensación de visitar espacios nuevos acumulando experiencias distintas.

En la última década se ha observado un crecimiento sustancial del número de turistas, con nuevos patrones de comportamiento de consumo turístico, en parte producidos como consecuencia de la evolución tecnológica, fenómeno al que, según Greztel et al. (2000), las empresas y destinos turísticos todavía no están preparados. Se trata del surgimiento de un nuevo paradigma turístico caracterizado por profundos cambios, tanto en la oferta como en la demanda, reflejo de una sociedad actual calificada condiversas designaciones: 
sociedad postfordista, sociedad de la información, sociedad del conocimiento, sociedad tecnológica o sociedad inteligente (Smart Society), (López Palomeque, 2015).

En este nuevo contexto, se precisan datos que permitan conocer el nuevo comportamiento del turista con el objetivo de planificar,en consecuencia, la actividad turística en particular y, el territorio en general.

Los datos, como sinónimo de información, constituyen la materia prima del conocimiento. Hoy en día éstos tienen un valor incalculable, hasta el punto de calificarse como "el petróleo del siglo XXI" (Mayer-Schönberger y Cukier, 2013), "una de las más preciadas mercancías" (Morozov, 2015), la base de la llamada cuarta revolución industrial o el cuarto paradigma de la sociedad científica actual, esto es, la ciencia orientada a la exploración de datos (Gray y Szalay, 2007; Hey et al., 2009; Gutiérrez, 2017). Así, su disponibilidad resulta fundamental en cualquier sector, siendo especialmente significativo en el turístico, en cuanto va a posibilitar la correcta toma de decisiones (Luque, Zayas y Caro, 2015).

Actualmente, las fuentes de información turísticas son múltiples. En este sentido encontramos las fuentes que recogen datos de los turistas procedentes de organismos públicos (Subdirección General de Conocimiento y Estudios Turísticos, Segittur, y el Instituto Nacional de Estadística, entre otros), fuentes privadas (Exceltur, Telefónica, BBVA) y fuentes de datos generadas por observatorios turísticos regionales, con una temporalidad irregular en dependencia de la innovación tecnológica y metodológica con la que se obtengan. Algunos de los factores que explican esta proliferación de estas fuentes son los siguientes (López Bonilla y López Bonilla, 2015): a) la transferencia de competencias en esta materia a las comunidades autónomas, generando nuevas demandas y creando institutos de estadística y centros de investigación propios; b) la integración en la Unión Europea; y c) los requerimientos de información de organismos internacionales, que reclaman estadísticas de mayor calidad y con mayor rapidez, por parte de los diferentes países.

Adicionalmente, en los últimos años, ha aparecido una nueva y valiosa fuente de datos: la que proporciona el denominado "Turista 2.0". Este nuevo turista, caracterizado por mantenerse continuamente informado, por la búsqueda de productos más personalizados y complejos, por un mayor nivel de exigencia y autonomía, y con nuevos hábitos de consumo, emplea un uso intensivo de la tecnología, hecho que convierte al turista tanto en receptor de información, como en generador de datos en relación a su comportamiento. En este sentido, la aparición de las nuevas tecnologías ha multiplicado los canales de recogida de datos referidos a los turistas, permitiendo conocer cuestiones que, hasta el momento, eran imposibles con las estadísticas tradicionales (Peñarrubia, 2018).

Dicho fenómeno, unido a la necesidad de la gestión y conocimiento de los territorios turísticos vinculada a la reciente revolución tecnológica acontecida, tiene como consecuencia el desarrollo de los denominados Destinos Turísticos Inteligentes (DTI), considerado por Ivars, Solsona y Giner (2016: 337) "no como un fin en sí mismo, sino como un marco de trabajo para adaptar la gestión turística a la economía digital, a la relevancia de las TIC en este sector y a la necesidad de reforzar la innovación y el conocimiento a la gestión turística", está siendo fundamental en la generación y uso de datos.

El DTI debe disponer de medios que permitan optimizar el uso de los datos en un entorno digital para obtener información actualizada sobre la evolución de la oferta y la 
demanda turística. En este sentido, la monitorización y digitalización de los destinos turísticos y de los turistas, ha generado un incremento exponencial en la producción de datos. Se trata de la "materia prima" que, convenientemente recogida, almacenada, procesada e interpretada, aporta el conocimiento que los actores turísticos necesitan para tomar decisiones adecuadas a las diferentes situaciones. Por ello se considera esencial la creación de un Sistema de Inteligencia Turística (SIT) que, en el caso de los DTI, se dirigen a facilitar la integración e interacción del visitante con el destino, mejorar su experiencia turística y, por ende, incrementar su satisfacción antes, durante y después del viaje. Por tanto, estos datos permiten el conocimiento del comportamiento del turista.

Así, y de acuerdo con Ivars (2017), "la inteligencia para un DTI consiste en seleccionar, conforme a sus características, los datos más valiosos para el gestor y empresariado local. Su análisis y gestión integrada en base a indicadores clave, permitirá obtener ventajas competitivas que sustenten líneas de acción inteligentes".

Los actores relacionados con el DTI determinan el tipo de datos que debe integrarse en el mismo, bien sea porque los producen, bien porque los demandan y emplean. Así, el modelo de datos debe responder a las exigencias de los tres actores que integran el destino: a) los gestores del destino, que deben tomar decisiones fundamentadas en datos convertidos en conocimiento; b) la empresa privada, como demandantes y generadores de datos; y c) los propios turistas como centro de la estrategia territorial y empresarial a los que se les debe mejorar la experiencia y garantizar su satisfacción.

No obstante, en la práctica, se observa que, aunque las actuales fuentes de información primaria en materia turística son numerosas, éstas no siempre se encuentran al alcance de la totalidad de los actores o la información accesible no dispone del contenido y calidad necesaria para la toma adecuada de decisiones.

En este contexto, y con el objetivo de proporcionar información que permita mejorar la "inteligencia" en el destino, resulta vital conocer la opinión de los actores públicos y privados en relación a cuestiones fundamentales como la identificación de los datos considerados relevantes para el análisis del comportamiento del turista, la validez de las actuales fuentes estadísticas públicas, su relación con las nuevas fuentes de datos privadas provenientes de las Nuevas Tecnologías de la Información y Comunicación (NTIC), así como la definición de las bases de una propuesta metodológica para la recogida de dichos datos.

\section{OBJETIVOS Y METODOLOGÍA}

En la medida que los expertos consideran imprescindibles los datos relativos al comportamiento del turista en procesos de toma inteligente de decisiones para la planificación y gestión territorial turística, la presente investigación pretende responder, en este sentido, a dos objetivos. Por un lado, elaborar un diagnóstico (evaluación de uso y necesidades) de dicha información y, por otro, determinar el grado de eficiencia y valía de este tipo de datos para los expertos.

La investigación parte de la hipótesis de que existe un gran volumen de datos respecto al comportamiento de los turistas. Sin embargo, ésta no es una información válida para la toma de decisiones inteligentes en los procesos de planificación estratégica y gestión 
de destinos turísticos. Asimismo, el enorme volumen de datos generados no se aprovecha para convertirlo en conocimiento.

Para ello se plantea la realización de entrevistas cualitativas personales a un grupo de expertos siendo, la mayoría de ellos, profesionales del ámbito geográfico regional de las Islas Canarias en cuanto se trata de uno de los principales destinos turísticos vacacionales de litoral de Europa; prueba de ello es que, según el Instituto Canario de Estadística (ISTAC), recibió 15.110.866 visitantes en el año 2019, ocupando el primer puesto en el ranking de pernoctaciones en establecimientos turísticos de no residentes de las 272 regiones NUTS 2 de la Unión Europea según EUROSTAT.

\subsection{El muestreo teórico: la selección del grupo de expertos}

Las diferentes fuentes de datos en materia turística existentes a distintas escalas y con distinta naturaleza permiten usos muy distintos en función del colectivo que los analice. La administración pública y la empresa privada turística son las principales generadoras de los datos. A su vez, junto a las instituciones académicas y centros de investigación, constituyen los tres actores principales que los utilizan e interpretan.

Este hecho determina la conveniencia del uso de la técnica de investigación cualitativa basada en entrevistas en profundidad individuales realizadas a un grupo de expertos. Esta técnica ha sido ampliamente utilizada en el campo de las ciencias sociales (Berg y Lune, 2014) y, en particular, en el turismo (Santana, 2005; Huete, Mantecón y Mazón, 2008; Anbalagan y Lovelock, 2014; Ponting y O’Brien, 2014; Rittichainuwat y Rattanaphinanchai, 2015; Mercado y Fernández, 2016).

En nuestro caso, el uso de esta técnica permitió la interacción dinámica, dialógica, personal y directa entre los investigadores y el experto, persiguiendo que exprese de manera detallada sus opiniones sobre el objeto de la presente investigación.

Somos conscientes de las principales desventajas de esta técnica: la interpretación subjetiva del entrevistador y la complejidad en el análisis de la información obtenida en la entrevista. No obstante, ambas debilidades se han compensado por el hecho de que ha sido posible conocer, con mayor profundidad y precisión, la información deseada para cada caso de estudio.

Las entrevistas se realizaron a expertos de carácter multidisciplinar y multisectorial relacionados estrechamente con la generación o uso de estadística pública turística procedentes del sector privado/público y ámbito técnico/universitario. Asimismo, todas las personas entrevistadas, además de estar estrechamente vinculadas a dichos organismos, son creadoras, usuarias o demandantes de las fuentes de datos objeto de evaluación tratándose, en definitiva, de los profesionales que reúnen mayores conocimientos en relación al tema de investigación.

Los expertos fueron cuidadosamente seleccionados para la investigación en función de su conocimiento sobre el tema objeto de estudio, cargo de responsabilidad, representatividad a diferentes escalas y sectores, y vinculación, y/o conocimiento, al ámbito geográfico en el que se desarrolla la investigación, las Islas Canarias. 


\section{Cuadro 1 \\ GRUPO DE EXPERTOS ENTREVISTADOS}

\begin{tabular}{|c|c|c|c|}
\hline $\begin{array}{l}\text { GRUPO DE } \\
\text { ESTUDIO }\end{array}$ & CARGO & ORGANISMO & ESCALA \\
\hline \multirow{4}{*}{ Universidad } & $\begin{array}{l}\text { Profesor Departamento } \\
\text { Sociología y } \\
\text { Antropología }\end{array}$ & Universidad de La Laguna & Nacional \\
\hline & $\begin{array}{l}\text { Profesor Departamento } \\
\text { Economía Aplicada }\end{array}$ & Universidad de La Laguna & Nacional \\
\hline & $\begin{array}{l}\text { Profesor área de Análisis } \\
\text { Geográfico Regional }\end{array}$ & Universidad de Alicante & Nacional \\
\hline & $\begin{array}{c}\text { Profesora Departamento } \\
\text { Sociología y } \\
\text { Antropología } \\
\end{array}$ & Universidad de La Laguna & Nacional \\
\hline \multirow{8}{*}{$\begin{array}{l}\text { Administración } \\
\text { Pública }\end{array}$} & Directivo & $\begin{array}{c}\text { Sociedad Estatal para la Gestión } \\
\text { de la Innovación y las Tecnologías } \\
\text { Turísticas (SEGITTUR) }\end{array}$ & Nacional \\
\hline & Coordinador de Proyectos & $\begin{array}{c}\text { Instituto Valenciano de Tecnologías } \\
\text { Turísticas (Invat.tur) }\end{array}$ & Regional \\
\hline & Jefe de Servicio & $\begin{array}{l}\text { Instituto Canario de Estadística } \\
\text { (ISTAC) }\end{array}$ & Regional \\
\hline & Jefa de Servicio & $\begin{array}{c}\text { Instituto Canario de Estadística } \\
\text { (ISTAC) }\end{array}$ & Regional \\
\hline & Directivo & $\begin{array}{l}\text { Factoría de Innovación Turística de } \\
\text { Canarias }\end{array}$ & Regional \\
\hline & Directiva & Promotur Turismo de Canarias & Regional \\
\hline & Directiva & $\begin{array}{c}\text { Turismo de Tenerife. Cabildo de } \\
\text { Tenerife }\end{array}$ & Insular \\
\hline & Responsable Unidad & Cabildo de Tenerife & Insular \\
\hline \multirow{6}{*}{$\begin{array}{l}\text { Empresa } \\
\text { privada }\end{array}$} & Directivo & $\begin{array}{c}\text { Instituto Tecnológico Hotelero } \\
\text { (ITH) }\end{array}$ & Nacional \\
\hline & Técnico & Noray Consultoría & Nacional \\
\hline & Directivo & Cadena Meliá Internacional & Nacional \\
\hline & Directivo & $\begin{array}{c}\text { Asociación Hotelera y } \\
\text { Extrahotelera de Tenerife, La } \\
\text { Palma, La Gomera y El Hierro } \\
\text { (Ashotel) }\end{array}$ & Regional \\
\hline & Directivo & Hoteles R2 & Regional \\
\hline & Directivo & Hotel Santa Mónica & Insular \\
\hline
\end{tabular}

Fuente: Peñarrubia, 2018.

Igual relevancia ha tenido la identificación de las personas a entrevistar a través del método bola de nieve. Así, cada persona entrevistada sugirió la entrevista de otras personas relevantes en cuanto que eran expertas en el tema en cuestión. 
Finalmente se realizaron 18 entrevistas (cuadro 1). Se trata de expertos "cualificados", en cuanto son usuarios que utilizan las estadísticas de forma habitual como instrumento para el desarrollo de su actividad profesional y/o investigadora siendo una representación de los principales tipos de usuarios y/o productores de estadísticas: técnicos de organismos de la administración pública, profesores universitarios, investigadores de prestigio, gerentes de patronales, así como personal y directivos de empresas privadas. Sus opiniones se encuentran estrechamente vinculadas al perfil académico de los entrevistados (economistas, sociólogos, geógrafos, estadísticos, psicólogos). Todos respondieron al cuestionario de manera proactiva y participativa.

Por último destacar que, aunque se ha entrevistado a un número relativamente reducido de expertos/as, la cantidad de información obtenida ha sido muy importante fundamentalmente por dos cuestiones: por una parte, las personas que han participado en esta investigación tienen un amplio conocimiento y experiencia en relación con el objetivo de la misma, en cuanto bien han participado en la creación de Sistemas de Información Turística (SIT), o usan habitualmente los datos estadísticos en los contextos de los actualmente denominados Destinos Turísticos Inteligentes (DTI).

Por otra parte, tras las entrevistas realizadas, se llegó a un "punto de saturación de la información”, en cuanto que las opiniones y comentarios comenzaron a repetirse. Así, consideramos que la información recogida resultó adecuada en relación con los objetivos de investigación, a la vez que se consideró que un mayor número de entrevistas no hubieran aportado una información cualitativa diferente, esto es, "nuevas entrevistas no hubieran añadido nada relevante a lo conocido" (Vallés 2009: 68).

\subsection{Características de cuestionario}

Los cuestionarios se confeccionaron mediante preguntas sobre hechos, comportamientos y opinión de tipo abierto. El fin ha sido que el entrevistado aportase una mayor información, fomentado, incluso, la aparición de nuevas ideas no contempladas inicialmente en la investigación. Sin embargo, este tipo de preguntas presenta la desventaja que la duración del tiempo de entrevista se alarga, los entrevistados pueden presentar mayor rechazo a responder sobre temas sensibles y, sobre todo, dificultades de registro, codificación y análisis de las repuestas. Asimismo, las preguntas se han planteado en batería proponiéndose cuestiones que permiten obtener información relacionada entre sí a partir de preguntas encadenadas y complementarias entre ellas.

Se han elaborado dos cuestionarios con enfoques similares, aunque con matices diferentes: uno para aquellos expertos de la empresa privada, y otro para los procedentes de la administración pública y el ámbito universitario. Los cuestionarios fueron diseñados como guion de la entrevista personal, con el objetivo de centrar el tema o problema objeto de estudio.

Cada entrevista se caracterizó por su flexibilidad y por el hecho que, aunque se articuló en torno a un cuestionario estructurado, éste se fue modificando durante la entrevista, eliminando cuestiones, en función de las respuestas que el entrevistado iba dando, o añadiendo otros detalles interesantes para la investigación. Así, la orientación de la entrevista semiestructurada quedó marcada por el propio entrevistado, más que por el entrevistador, 
con el fin de descubrir no sólo los aspectos más objetivos de la investigación, sino también las opiniones más personales y subjetivas de cada experto. Además, el uso de este tipo de herramienta cualitativa nos permitió obtener información más compleja al relacionarse con aspectos más cercanos al subconsciente del individuo, a la psicología de las personas, dominadas por aspectos como valores, impulsos, emociones y motivaciones, aspectos que no siempre pueden ser expresados en un cuestionario (López Bonilla y López Bonilla, 2015).

Las preguntas se organizaron de manera secuencial y se estructuraron en seis bloques para el cuestionario de los expertos de la administración pública y los expertos universitarios, y en cinco el cuestionario planteado a los técnicos de la empresa privada. Los bloques quedan claramente diferenciados conduciendo al entrevistado, en ambos casos, hacia una maduración del tema y una senda argumental que, finalmente, permitía al entrevistado emitir una valoración-reflexión en la pregunta formulada en la última parte de la entrevista. Las preguntas de cada bloque se encuentran relacionadas entre sí comenzando por cuestiones más generales y concluyendo con aquellas más específicas.

Los bloques en los que se ha estructurado la entrevista para la administración pública y la academia serían los siguientes:

1. El nuevo consumidor como centro de la estrategia empresarial turística

2. La administración pública como generadora de datos

3. Los datos de la empresa privada

4. Las empresas turísticas como demandantes de datos

5. El nuevo enfoque de los Destinos Turísticos Inteligentes

6. ¿Existe un Sistema de Inteligencia Turístico público (SIT)?

Mientras que los bloques en los que se ha estructurado la entrevista para la empresa privada serian similares, aunque, con matices diferentes:

1. El nuevo consumidor como centro de la estrategia empresarial turística

2. Las empresas turísticas como generadoras de datos

3. Las empresas turísticas como demandantes de datos

4. El nuevo enfoque de los Destinos Turísticos Inteligentes

5. ¿Qué aportaría la información recogida de su empresa a su Sistema de Inteligencia Turístico público (SIT)?

\subsection{La sistematización de la información obtenida de la entrevista personal}

Siguiendo a Miles y Huberman (1994), Rubín y Rubín (1995) y Álvarez-Gayou (2003), la sistematización de la información obtenida de la entrevista personal siguió el siguiente proceso secuencial:

1. Obtención de la información. Mediante cita previa, y tras explicar el motivo de la entrevista y el objeto de estudio, se concertaron las entrevistas. Cada una de ellas se celebró, a propuesta del entrevistado, en aquellos entornos que consideraron más favorables, realizándose todas ellas en Tenerife, Gran Canaria, Madrid y Valencia.

Las entrevistas fueron personales e individuales a cada experto, en los meses de diciembre de 2017 y, febrero y abril de 2018. La duración media de la entrevista fue de 45 minutos. No obstante, se observaron importantes diferencias en la duración de la entrevista 
que se debieron, principalmente, al interés personal del entrevistado por el tema objeto de estudio; así, mientras alguna entrevista se resolvió en 20 minutos, otros entrevistados necesitaron dos horas de reflexión.

2. Captura, transcripción y ordenación de la información. Previo consentimiento de los entrevistados, las conversaciones fueron grabadas a través de registro electrónico, generando un archivo de audio por cada entrevista, así como el registro en papel de algunas notas manuscritas sobre aspectos relevantes comentados durante la conversación. Posteriormente, los investigadores escucharon las conversaciones mantenidas en los archivos de audio y transcribieron la información obtenida a archivos en formato Word para poder comenzar su análisis.

3. Codificación de la información. La información obtenida se agrupó en categorías que concentraban las ideas, conceptos y temas similares que iban siendo descubiertos por los investigadores. La agrupación y ordenación de la información comenzó a sentar las bases para la elaboración de conclusiones.

4. Procesamiento de la información. El análisis de las citas textuales permitió identificar un conjunto de premisas básicas vinculadas con el tema de investigación. Se trató de identificar todas las cualidades posibles para alcanzar un entendimiento en profundidad. Una vez encontrados conceptos y temas individuales, se relacionaron entre sí para poder elaborar una explicación integrada. Así pues, dentro del proceso, se distinguen dos fases: mientras en la primera se procedió al análisis de la información dentro de cada categoría, en la segunda se comparó entre las mismas, buscando los vínculos que puedan existir entre ellas. Finalmente se relacionaron las categorías obtenidas en el paso anterior entre sí con los fundamentos teóricos de la investigación.

\section{RESULTADOS}

\subsection{Conveniencia y uso de las estadísticas turísticas para la administración pública}

El grupo de ocho expertos seleccionados para conocer la opinión sobre la conveniencia y uso de las estadísticas públicas en la administración pública se ha subdividido en tres grupos: a) el integrado por tres responsables de organismos que elaboran fuentes estadísticas públicas en las Islas Canarias; b) el integrado por dos responsables de los de organismos públicos que emplean las estadísticas públicas para la promoción turística de las Islas Canarias a distintas escalas; y c) el integrado por tres responsables de las instituciones que utilizan datos estadísticos de distinta naturaleza para la gestión e innovación de los destinos turísticos a escala regional (Islas Canarias y Comunidad Valenciana) y escala nacional.

El cuestionario parte del interés por conocer la opinión de la administración pública por el valor del consumidor o turista. Los ocho entrevistados coinciden de manera unánime en que la administración pública vuelca sus esfuerzos en el conocimiento del turista, siendo plenamente consciente de su importancia. Algunas de las posturas más críticas se refieren a que la identificación de las necesidades del turista persigue la mercantilización de sus intereses. 
Consideran que las empresas privadas y, especialmente, la administración pública, utiliza con regularidad las fuentes de datos públicas para tomar decisiones. El sector turístico ha tardado en asumir la necesidad de conocer al turista, al existir la figura del turoperador que, tradicionalmente ha sido el cliente de los destinos. Esto varió con el mencionado cambio en la consideración del turista, pasando a ser el centro de las decisiones. Ello modificó la estrategia de instituciones como PROMOTUR (Promotur Turismo Canarias, S.A. es la sociedad mercantil pública adscrita a la actual Consejería de Turismo, Cultura y Deportes del Gobierno de Canarias), en cuanto que el enfoque y la organización de la empresa se dirigió al conocimiento final del turista. Ello contribuyó a que otros organismos públicos también reorienten los enfoques y el diseño de los cuestionarios, induciendo a la inclusión de cuestiones impensables hasta ese momento.

Desde Promotur y el Cabildo de Tenerife apuntan que, aunque dirigirse al turista y conocerlo con el objetivo de satisfacer sus necesidades y expectativas turísticas resulta ineludible, no conviene descuidar y desatender las necesidades del propio destino turístico. Para ello consideran necesario valorarlo en su totalidad, reflexionando y defendiendo el ajuste que debe existir entre el tipo de demanda existente (turista) y las características del propio territorio como destino turístico. En este sentido, el conocimiento del turista para su adaptación al territorio resulta esencial, empleando la segmentación y la definición de patrones de comportamiento como técnica de cribado para la selección del turista.

Por lo que se refiere a la función de generación de datos por parte de la administración pública, los diferentes actores consideran que las necesidades de información detectadas a consecuencia de los cambios acontecidos en el sector turístico y, más concretamente, en el cambio del comportamiento del turista, obligan a crear nuevos datos. Así, las entidades de promoción explican cómo éstos son el motor del cambio, al plantear las necesidades de datos a los organismos públicos de estadística. En este sentido, los organismos de estadística confirman el hecho que, al tratarse de procesos estadísticos sometidos a legislación y metodologías rigurosas, no resulta fácil realizar cambios, al menos, de una manera inmediata.

Independientemente de quien impulse los cambios en la recogida e identificación de los datos que deben incorporarse en las nuevas estadísticas, el hecho es que desde el año 2008, los organismos estadísticos competentes como el Instituto Canario de Estadística (ISTAC) y Cabildo las han asumido demostrando la existencia de una voluntad real de cambio. Prueba de ello son las modificaciones realizadas en los últimos cuestionarios a finales de 2017 y principios de 2018, así como las nuevas operaciones en esta línea de investigación turística contempladas en el Plan Estadístico de Canarias 2018-2022 aprobado el 21 de mayo de 2018 por el Consejo de Gobierno de Canarias.

Los datos que se recogen actualmente no son diferentes a los anteriores. Se trata de hacer lo mismo, pero con niveles de profundidad distintos en cuanto a escala (difundiéndose en la actualidad a escala de microdestino) y tipo de información, así como la adaptación de los instrumentos metodológicos a las necesidades detectadas, con el fin de hacerlos más potentes. A pesar de esta opinión, se considera que se han acometido cambios sustanciales, desde el punto de vista de las variables, que ahora se han incluido en los nuevos cuestionarios, tanto las elaboradas por el ISTAC como por el Cabildo; de este modo, se han incluido cuestiones relativas a la motivación, gastronomía, medio ambiente, 
movilidad, teletrabajo, necesidades y usos del wifi, percepciones de zonas comerciales abiertas, carreteras, playas, así como infraestructuras y servicios próximos a los establecimientos hoteleros.

En cuanto a los canales de recogida y frecuencia de los datos, se distingue entre los datos que provienen de la estadística pública, y los que se emplean en los organismos de gestión y promoción del destino, compuestos, tanto por las estadísticas públicas, como por información procedente de redes sociales, páginas web y del propio sector privado (en especial de datos hoteleros), así como información cualitativa a partir de técnicas de focus group, debates a expertos o estudios ad hoc.

Todos los expertos coinciden en que, en la medida que a cada uno se le permite, hacen una difusión correcta y extendida de los datos. Sin embargo, reconocen que, en muchas ocasiones, el resto del sector le exige una mayor interpretación de los datos o su presentación a modo de cuadro de mandos, con el objetivo de simplificar el análisis. Al mismo tiempo, indican que la satisfacción con la información generada es una cuestión completamente subjetiva que depende en gran medida del tipo de usuario.

La elaboración de las estadísticas públicas implica una serie de problemas derivados de la recogida y difusión de los datos. Los principales problemas se derivan de los permisos necesarios en aeropuertos para realizar las encuestas, la selección de los vuelos, la coordinación con múltiples empresas privadas que, a su vez, también realizan cuestionarios de opinión extraoficiales, la desmotivación por parte de los turistas para contestar a los cuestionarios, dejándolas en muchas ocasiones a mitad, así como los errores al contestar preguntas que no llegan a ser entendidas. Estos problemas obligan a que el número de cuestionarios totales realizados en cada operación deba multiplicarse por tres para poder obtener una muestra significativa.

Las opiniones relativas a los datos generados por la administración pública susceptibles de posibilitar el conocimiento del turista se fragmentan atendiendo a quien los crea y quien las usa. ISTAC y Cabildo consideran que son suficientes, que se difunden de manera adecuada y a las escalas con mayor detalle posible, así como que cumplen las exigencias mínimas de los agentes privados. Esta opinión es contraria a las entidades que las utilizan, al considerar que "todavía falta mucho por saber", considerando especialmente relevante la mejora del conocimiento de las motivaciones.

Esta diferencia de opinión, ante un mismo hecho, podría justificarse por el momento de inflexión en el que se encuentran las estadísticas Canarias. Las instituciones estadísticas competentes consideran que responden a las necesidades de información del sector y han modificado los cuestionarios en base a las mismas; por ello estiman que los datos que ya se han empezado a recoger poseen un gran valor. Por el contrario, las entidades que aún los usan no constatan los resultados de este cambio y continúan juzgando en base a los datos convencionales que hasta el momento se han venido haciendo $\mathrm{y}$, por tanto, anteriores a los nuevos cuestionarios realizados a partir de enero de 2018.

Todos los entrevistados sostienen el mismo discurso en relación con el uso de los nuevos datos que genera la empresa privada: no hay que obviarlos ya que son muy interesantes, aunque tienen un coste de adquisición muy alto, están muy sesgados y se desconoce la metodología de elaboración, lo que cuestiona su fiabilidad y uso. Desde Promotur explican que "son tantas las que les ofrecen a diario que te paralizas y de momento no se 
ha tomado la decisión de comprar ninguna a consecuencia de los inconvenientes mencionados". Consideran de gran interés la información que se encuentran en las bases de datos de transacciones económicas mediante tarjetas de pago a través de los terminales de punto de venta (TPV) de la entidad bancaria BBVA. Lo mismo sucede con las de las operadoras telefónicas (Orange y Telefónica), que, en muchos casos, únicamente venden un servicio -y no los propios datos-, con el inconveniente de la existencia de zonas de sombra y mercados con los que no trabaja. Los datos de la empresa privada de reservas mundiales de viajes AMADEUS también son interesantes (cuyos datos de un año doblan el coste de la elaboración anual de la Encuesta de Gasto turístico del ISTAC), los datos de sensorización de empresas de alquiler de vehículos en el destino para conocer detalles de las rutas, los de Tripadvaisor, Edreams, datos sobre la reputación del destino y del comportamiento del turista en Redes sociales, e incluso, los propios datos que ofrece Google Analytics.

Si bien los datos tradicionales utilizados hasta el momento han permitido tomar buenas decisiones, ahora la forma de interactuar con el turista es distinta y da lugar a nuevas fuentes de información. Por ello, conviene adaptarse al nuevo contexto, necesidades de datos y procedimientos de recogida. En este sentido, la administración pública no está cerrada o es ajena a las necesidades del resto de actores. Sin embargo, no es posible dejar de hacer estadísticas públicas, ya que el Estado debe tener un producto estadístico oficial que sea estable a lo largo del tiempo y que genere confianza. Ello no es óbice para que la administración pública genere nuevos sistemas de recogida de datos a partir de la sensorización del destino; se trata de una acción que permite obtener datos con múltiples objetivos, así como desarrollar mecanismos de colaboración con aquellas empresas privadas que generan datos de mayor valor y que el Estado no es capaz de generar. En este sentido, ambas fuentes de datos, estadísticas públicas y datos privados son complementarias y actualmente ya se han formalizado acciones en ésta línea para la elaboración del futuro Padrón de Habitantes 2021.

A pesar de que el término DTI es un concepto, en origen, vinculado a la administración, sorprende el hecho de que la mayoría de los actores desconocen su verdadero significado. Además, los que afirman conocerlo suelen relacionarlo con un componente meramente tecnológico. Del mismo modo, a excepción de la Sociedad Mercantil Estatal para la Gestión de la Innovación y las Tecnologías Turísticas, S.A.M.P. (SEGITTUR), que lo considera como una nueva forma de gestionar la interactuación con los visitantes, y el Instituto Valenciano de Tecnologías Turísticas (Invat·tur) que lo considera como un nuevo modelo de trabajo en cuanto a la gestión del destino basado en la gobernanza y que emplea la tecnología como herramienta al servicio del destino, el resto, no consideran que sea un término adecuado por el excesivo abuso. La difusión del término, en muchas ocasiones comunicado en relación con la tecnología, hace que se vea como una moda que será pasajera. Asimismo, consideran que lo "Smart" es únicamente una herramienta, mientras que la inteligencia en el destino se consigue por parte de las personas.

Por último, en relación con la construcción de un sistema de información pública mediante colaboración pública-privada, todos los entrevistados consideran que sería una iniciativa extraordinaria y que ya se está produciendo el proceso, al menos por parte de la administración pública. A pesar de ello, consideran que las empresas privadas no son favorables a aportar sus datos, pues permitiría desvelar sus estrategias. En este sentido, desde 
el ISTAC afirman que este sistema ya existe, aunque no desde una perspectiva formal, con cabildos y universidades; a este respecto, interpretan que un SIT debería ser un conjunto de alianzas entre el sector público y el sector privado que permita identificar y satisfacer necesidades para incorporarlas en una estrategia de análisis y que, a pesar de tratarse de un proceso complicado, opinan que entre estos organismos se está consiguiendo.

\subsection{Conveniencia y uso de las estadísticas turísticas para la empresa privada}

Para analizar la conveniencia y uso de los datos estadísticos relativos al comportamiento del turista por parte de la empresa privada, se han realizado seis entrevistas. La mayoría de ellas han sido personas relacionadas con los establecimientos hoteleros, dado el peso que tienen los establecimientos alojativos reglados, así como por el papel destacado que tienen en los procesos de aporte de datos a la estadística pública. Así, se realizaron entrevistas a personas destacadas de cadenas hoteleras de diferentes dimensiones y por tanto con visiones de escala distintas: de la Cadena Meliá Hotels International (con más de 350 hoteles en 40 países), de la cadena Hoteles R2, con seis hoteles en España, así como el responsable del hotel independiente Santa Mónica Suites de categoría 4 estrellas superior ubicado en Maspalomas (Gran Canaria). También se han entrevistado a personas pertenecientes a entidades con diferentes implicaciones en el sector turístico privado: un centro de innovación, adscrito a la Confederación Española de Hoteles y Alojamientos Turístico, el Instituto Tecnológico Hotelero (ITH), la patronal de alojamiento turístico de la provincia de Santa Cruz de Tenerife y una empresa de construcción de software para hoteles.

Las opiniones vertidas son tan coincidentes como opuestas. Éstas revelan las distancias existentes en el sector privado con respecto a las necesidades de información por parte de la estadística pública, a la vez que la capacidad de generar su propia información respecto al conocimiento del propio turista, entendido, en este caso, como un cliente.

Todos los entrevistados están conformes con la idea de que el turista ocupa ahora el centro de las empresas turísticas, siendo su análisis segmentado y opinión las que vertebran y dan lugar a las estrategias empresariales. Indican que el nuevo comportamiento del turista en la contratación del viaje ha comportado un cambio del cliente final de los establecimientos de alojamiento turístico. Este hecho ha determinado la necesidad de proceder al conocimiento de su comportamiento, en detrimento del turoperador, su principal cliente hasta ese momento.

La generación de datos por parte de las empresas depende fundamentalmente de las inquietudes que motiven el conocimiento de los clientes y de las capacidades y recursos disponibles. Los datos de interés son, entre otros, los relativos a las motivaciones, las experiencias y el valor económico real o potencial, y, por tanto, aquellos generalmente recogidos en los propios CRM de las empresas a través de cuestionarios de satisfacción. En particular se observa que el interés por la recogida de datos y la exigencia de variables para un mayor conocimiento del turista es superior en la cadena de hoteles de mayor tamaño y desciende a medida que el hotel se individualiza. Así destacan las siguientes variables analizadas (cuadro 2) en función del tipo de hotel. 
Prueba de la importancia del conocimiento del cliente para la cadena Meliá es que en los últimos años han pasado de tener 18 millones de datos a 20 billones en relación a su comportamiento.

\section{Cuadro 2 \\ RELACIÓN ENTRE EL TIPO DE ALOJAMIENTO TURÍSTICO Y LA RECOGIDA DE DATOS}

\begin{tabular}{|c|c|c|}
\hline $\begin{array}{c}\text { Tipo de } \\
\text { alojamiento }\end{array}$ & Variables consideradas & Medio \\
\hline $\begin{array}{c}\text { Gran Cadena } \\
\text { Hotelera }\end{array}$ & $\begin{array}{l}\text { - Transaccionales: reservas según el tipo de hotel, } \\
\text { recurrencia de compra, viene con niños, viaja } \\
\text { solo, viaja a qué destinos. } \\
\text { - Demográficas: años que tiene, de dónde es, de } \\
\text { qué lugar viene. } \\
\text { - Psicográficas: cruzando la dirección postal con } \\
\text { bases de datos de variables que indican los perfi- } \\
\text { les psicográficos del cliente en su entorno } \\
\text { - Performance de cliente: a través de cookies, } \\
\text { comportamiento en la web, qué hacen, que tipo } \\
\text { de páginas visitan, qué amigos tienen; a través } \\
\text { de las redes sociales, intereses, hobbies, llama- } \\
\text { das al contact center y qué dice. } \\
\text { - Geolocalización de personas para campanas vía } \\
\text { geolocalización. }\end{array}$ & $\begin{array}{l}\text { - Cualquier interac- } \\
\text { ción con el cliente } \\
\text { (llamadas, web, } \\
\text { etc.) } \\
\text { - Cuestionarios on } \\
\text { line } \\
\text { - Reputación en redes } \\
\text { Sociales }\end{array}$ \\
\hline $\begin{array}{c}\text { Mediana Cade- } \\
\text { na Hotelera }\end{array}$ & $\begin{array}{l}\text { - Satisfacción general } \\
\text { - Motivaciones } \\
\text { - Experiencias }\end{array}$ & $\begin{array}{l}\text { - Encuestas Satisfac- } \\
\text { ción } \\
\text { - Reputación en redes } \\
\text { Sociales }\end{array}$ \\
\hline $\begin{array}{l}\text { Hotel Indepen- } \\
\text { diente }\end{array}$ & - Expectativas & $\begin{array}{l}\text { - Encuestas Satisfac- } \\
\text { ción } \\
\text { - Relaciones Públicas } \\
\text { - Reputación en redes } \\
\text { Sociales } \\
\text { - Plataforma Review } \\
\text { Pro }\end{array}$ \\
\hline
\end{tabular}

Fuente: Peñarrubia, 2018. 
Entidades como Ashotel son conscientes de que se debe mejorar la estrategia de obtención de datos del cliente alojados en establecimientos turísticos ya que, una vez que el cliente llega al alojamiento, éste dispone de varios días para poder consultarle por diferentes medios: las denominadas "encuestas de satisfacción”, las reuniones, los servicios gratuitos con el objetivo de testar productos, etc. Así, se obtienen una serie de datos que podrían ser recompensados mediante contraprestaciones gratuitas por parte del hotel. Esta recogida de datos se hace principalmente por parte de aquellas cadenas hoteleras que disponen de recursos, de modo que, su departamento comercial, a medida que incrementa la disponibilidad de datos, se convierte en el área de inteligencia que aporta el conocimiento necesario para la toma de decisiones.

En cuanto a la redundancia de operaciones estadísticas a la que están sometidos los establecimientos hoteleros, únicamente el gerente de Ashotel hace mención, de manera muy superficial, a esta cuestión. Además, se plantea el hecho que, aunque las empresas necesitan datos para la toma de decisiones, también necesitan inmediatez y previsión, mientras que los datos que las estadísticas públicas generan sólo son de tipo histórico, de modo que no permiten observar tendencias, y es por este motivo por lo que el sector evita emplearlas al no cubrir las necesidades de demanda de datos de carácter predictivo.

En relación con la demanda de datos por parte de la empresa privada, las visiones y comportamientos son muy distintas dentro del mismo colectivo. Se observa que, dependiendo de las dimensiones de los hoteles (independientes o en cadenas hoteleras), la necesidad de información y su uso varía considerablemente. Llama la atención que, siendo todos conscientes de la necesidad de obtener datos sobre el turista para planificar estrategias, las grandes cadenas hoteleras no usan ningún tipo de estadística pública, empleando los datos producidos por su propio CRM. Así, consideran que disponen de mucha más información, con más detalle y con carácter predictivo de la que poseen y publican los propios institutos de estadística. Por ello, este tipo de información no les resulta, en absoluto, útil, al considerar que aportan datos demasiados agregados en un momento que emerge un modelo que obliga a la personalización. En cuanto a las empresas hoteleras de tamaño mediano, apuestan por el uso de los datos que generan los Institutos regionales de Estadística a pesar de su escasa desagregación territorial, así como el apoyo de la información cualitativa obtenida por los cuestionarios de satisfacción. El hotel independiente se nutre de los datos habitualmente aportados por el Patronato de Turismo, la información recogida en el propio establecimiento y la situación del mercado a partir de reuniones de proximidad con otros hoteleros. En este sentido, el resto de entrevistados, tomando como ejemplo el ITH, consideran que para las adquisiciones de hoteles si se consultan datos oficiales del INE; no sucede lo mismo en relación con el conocimiento del comportamiento del turista para el que se sirven de su propia información y del "olfato".

Cuando se pregunta en relación con los datos que debería generar la administración pública para que cubriera las necesidades de demanda de la empresa privada turística, las respuestas son variadas. Por una parte, están los que consideran imposible generar ningún dato que aporte algo nuevo, ya que los que necesitan se caracterizan fundamentalmente por estar desestructurados y por ser cualitativos, siendo imposibles de recoger en la actualidad por un instituto de estadística. Otros se plantean que los datos disponibles son suficientes, aunque se está haciendo un uso insuficiente de los mismos. Un tercer tipo de opinión es 
que los datos debían tener una mayor desagregación territorial y ser presentados de manera mucho más visual, a modo de cuadro de mandos o widgets en la página del hotel con el objetivo de poder hacer una interpretación más rápida y eficiente.

En cuanto al nuevo enfoque de los Destinos Turísticos Inteligentes, se observa un desconocimiento casi absoluto de esta cuestión. Si bien tanto la persona entrevistada de la empresa de software, como los responsables de los hoteles, independientemente de su tamaño, desconocen por completo el concepto, por su parte, Ashotel y el ITH poseen un mayor conocimiento del concepto, destacando que éste último lo conoce en profundidad, concibiéndolo como un sistema de gestión de destino en el que la tecnología desempeña un papel prioritario. Únicamente las entidades privadas que tienen relación con la administración pública son conocedoras del término, no afectando ni considerándose incluidas en el mismo las empresas privadas hoteleras.

En cambio, sí existe un total acuerdo en el reconocimiento de que la tecnología es vital para el conocimiento del turista actual. Su utilización se considera conveniente en la medida que el consumidor está cada vez más informado dando lugar a una mayor transparencia al mercado. Así, consideran, en términos económicos, que la tecnología pone a la oferta y la demanda en un entorno más competitivo que permite que todas las empresas sean capaces de acceder al mismo cliente. No obstante, se reconoce la existencia de un sesgo, al no ser frecuente el uso de las tecnologías por parte de las personas de mayor edad.

Los medios considerados para la obtención de datos por medio de la tecnología son muy amplios. Los más utilizados son las redes Sociales (motivaciones y reputación) y el análisis de las cookies como rastro digital del comportamiento del turista en la navegación por la web. Por su parte, la recogida de datos en los establecimientos alojativos se produce a través de las llaves/tarjetas de proximidad (el internet de las cosas, IOT), que permiten monitorizar el comportamiento del cliente dentro del mismo.

Por último, resulta interesante la respuesta acerca de la disposición a incorporar sus datos a un SIT público. Si bien de manera unánime y en un primer momento responden de manera negativa, seguidamente reconsideran la respuesta y reflexionan sobre las ventajas que podría reportarles generar datos en un entorno colaborativo, donde pudieran compararse por productos y ámbitos territoriales, a la vez que les permitiera mejorar comportamientos. Tras su propio análisis cambian de opinión argumentando que lo harían siempre que se haga de manera que pueda repercutirles en un beneficio percibido. En esta línea, Ashotel ha propuesto, recientemente, la posibilidad de la creación de un Cloud Hotelero con el objetivo de almacenar y sistematizar los datos de los diferentes establecimientos de alojamiento turístico de la provincia de Santa Cruz de Tenerife; en este sentido, una buena parte han manifestado su interés por implementarlo, aprovechando la oportunidad de disponer de una infraestructura adecuada como es el ordenador ALIX (varios servidores en serie), considerado el segundo ordenador más potente de España.

\subsection{Conveniencia y uso de las estadísticas turísticas para fines de investigación uni- versitaria}

El tercer ámbito que emplea los datos de manera crítica y continua es el ámbito universitario que, desde la investigación, se convierte en parte activa en la aportación y transfe- 
rencia de conocimiento susceptible de ser utilizada en la toma de decisiones relativas a la planificación y gestión de los destinos turísticos. En este sentido, se han seleccionado tres perfiles disciplinares distintos: la Geografía, la Economía y la Sociología, con dos entrevistados. Son investigadores de reconocido prestigio, especializados en temas turísticos.

Todos tienen una conciencia general y una visión compartida sobre la consideración del turista como centro de la estrategia empresarial turística. Asimismo, destacan la importancia del turista y de su comportamiento en el territorio, al tiempo que opinan que la planificación territorial siempre tiene que tener en cuenta al turista. Del mismo modo, se considera que la investigación ha evolucionado de manera paralela al propio comportamiento del turista.

Se considera que tradicionalmente se ha potenciado, en mayor medida, las estadísticas de oferta en detrimento de las de demanda. Ello explica, por ejemplo, la no inclusión hasta hace relativamente poco tiempo, del motivo de la visita en muchas de las estadísticas públicas, lo cual ha obligado a solicitar los análisis motivacionales a consultoras privadas que realizaban informes y que aportaban una serie de resultados en los que no se explica la metodología.

Existe un consenso general acerca del papel de la administración pública como generadora de datos. Se considera que la administración pública genera desde hace unos años una mayor cantidad de datos, de mejor calidad, más sistemática, más accesible y con mayor transparencia. Sin embargo, se tiene la percepción que, exceptuando algunos institutos regionales que han incluido mayores desagregaciones territoriales y variables relativas a redes sociales, no se han incorporado nuevas variables para el análisis. Además, existen carencias importantes en los datos generados por la administración pública relacionados con los siguientes aspectos:

- La democratización y apertura de los datos turísticos, aumentando la transparencia y permitiendo el acceso al sector a los microdatos mediante Open Data.

- La inclusión de nuevas variables relacionadas con el nivel de estudios, motivaciones y comportamiento del turista en el destino.

- La necesidad de una mayor desagregación territorial a escalas subnacionales, e incluso locales.

- La necesidad de una mayor desagregación en los datos.

- La difusión de los datos en cuanto a tiempos y formas. Éstos deberían presentarse de manera más atractiva, visual e intuitiva, a modo de cuadro de mandos que incluyeran indicadores útiles al sector, en vez de presentar los datos en innumerables hojas Excel.

- Datos sobre redes sociales, y otras herramientas tecnológicas, que permiten conocer, con más detalle, el comportamiento del turista y su movilidad en el destino.

- Falta de recursos para la elaboración de las estadísticas.

- Retrasos en algunas publicaciones y cambios metodológicos que alteran las series históricas y recortes en las muestras.

- La necesidad de conocer las multimotivaciones y las multiexperiencias que, en la medida en que son subjetivas y cualitativas, resultan muy difíciles de abordar desde la perspectiva de los institutos de estadística, productores de información cuantitativa. 
- La duplicidad en las operaciones estadísticas turísticas que recaen sobre el sector hotelero, sometiéndoles a un proceso de redundancia de datos.

- Existe una sobrevaloración del dato. Se considera que no todos son necesarios y que convendría seleccionar un conjunto de variables realmente útiles para el análisis del comportamiento del turista.

- Aunque existe una gran cantidad de datos por parte de la administración pública, resultan insuficientes para conocer el comportamiento del turista.

Los datos producidos por las empresas privadas, bien digitales derivados de la huella o rastro que deja el uso de sistemas tecnológicos, bien producidos por empresas del propio sector (datos de los turistas recogidos por los propios hoteles), se consideran imprescindibles, siendo "una mina de información" que no puede obviarse. Sin embargo, de manera general, los investigadores desconfían de la generación de estadísticas privadas al carecer de la conveniente publicación de las metodologías y las muestras empleadas para llevarlas a cabo. Además, plantean la necesidad de reflexionar en torno a tres cuestiones: a) se trata de una información propia de una empresa, que, en algunos casos, supone el descubrimiento de una nueva línea de negocio, con lo que implica un alto coste de adquisición para empresas y administraciones; b) los datos que se comercializan son los que los propios usuarios han generado (sin ningún tipo de remuneración por parte de las empresas hacia los mismos) y con los que las empresas obtienen un beneficio; y c) habrán que evaluarse los efectos de la entrada en vigor el 25 de mayo de 2018 el Reglamento general de protección de datos, que establece normas comunes sobre el derecho de las personas a saber qué se hace con sus datos y los criterios para borrar, rectificar o suspender su tratamiento.

En la medida que estos datos complementan a los tradicionales públicos, se trata de buscar fórmulas que permitan incorporar las nuevas fuentes privadas a los destinos, sin coste por parte de la administración, a través de mecanismos colaborativos o de intercambio, a la vez que se desarrollan los procedimientos e instrumentos necesarios para adecuar las metodologías de la generación de datos privados a las de los datos estadísticos públicos, siguiendo los procesos de estándares de calidad previamente definidos. Asimismo, se considera que la estadística pública debe dar un salto con implicaciones legislativas que permitan la apropiación de los datos privados y su incorporación a los datos existentes. No obstante, esta acción de armonización no está exenta de ciertas inquietudes por parte de los diferentes institutos de estadística a consecuencia de la interrupción de nuevos datos en la continuidad de las series estadísticas. A pesar de los beneficios que reportaría al destino la unificación de ambos tipos de fuentes de datos (estadísticas públicas y fuentes de datos privadas), los investigadores apuntan, en caso de producirse esta situación, la complejidad que supondría el proceso de vertebración entre ambos intereses.

En cualquier caso, conviene analizar el uso y conveniencia de estas fuentes privadas y su necesidad en cada uno de los destinos, midiendo en términos de rentabilidad si el coste que implica justifica los beneficios de su utilización. En este sentido, se plantea el desigual coste de las distintas fuentes de datos generadas por las empresas privadas: mientras que las elaboradas por parte de empresas vinculadas a operadoras telefónicas o entidades bancarias, suponen un alto coste para los destinos, a la vez que, en caso de no ser cedidas a la administración, deben emplearse únicamente en acciones más puntuales, las generadas 
por las redes sociales, aplicaciones móviles, páginas web o la propia sensorización del destino, generan un importante rastro digital y presentan un acceso más fácil y económico que las anteriores. Evidentemente, el uso de una u otra vendrá justificado por el ajuste a los objetivos de cada investigación.

Se considera que muy pocas empresas turísticas emplean datos estadísticos públicos para la toma de decisiones, a pesar de que la estadística pública podría aportarles una perspectiva global del destino turístico. Estiman que los principales usuarios de estas estadísticas son las asociaciones hoteleras y ocasionalmente, grandes cadenas de establecimientos alojativos. No obstante, en la medida que no cubren sus necesidades de demanda de datos predictivos por tratarse de datos históricos, éstos satisfacen sus necesidades de información con las fuentes proporcionadas por sus proveedores digitales dedicados a la comercialización del producto en la web o las generadas por ellos mismos a partir de sus CRM. En cualquier caso, los investigadores entrevistados consideran que todavía no ha calado lo suficiente la cultura del uso del dato en las empresas, motivo por el que no son capaces de obtener el máximo rendimiento a los datos existentes.

Al reflexionar acerca del paradigma de los DTI, las visiones de los investigadores varían considerablemente distinguiendo dos perfiles: los especializados en los mismos y los que no han desarrollado su actividad en este campo, aunque han oído hablar del concepto. Los primeros son conscientes de que el sector privado desconoce el término, atribuyendo este hecho a la vinculación del término a la gestión estratégica del destino por parte de la administración pública; en este sentido, consideran el concepto desde un enfoque más amplio que pone a la gobernanza en el centro de la estrategia de los DTI, a la vez que sostienen que suponen para los destinos un concepto de gestión global y colaborativo que proporciona una sistemática de trabajo apoyada en instrumentos que permiten conocer y satisfacer la demanda. Los segundos, lo consideran un término "de moda", considerando que, si alude al hecho de planificar los destinos turísticos en base al empleo de datos, entonces "muchos son inteligentes desde hace mucho tiempo".

El uso de la tecnología se considera fundamental para el conocimiento del turista debiéndose a que se hace un uso habitual y continuado de la misma en tareas cotidianas como comprar, informase, reservar, etc., dejando una importante huella digital durante el proceso. Sin embargo, la tecnología se usa de manera desigual en cada destino turístico, por lo que su utilización debe quedar condicionada a la adaptación a entornos geográficos turísticos y a las necesidades reales de tecnología, no siendo válido el mismo patrón de comportamiento en todos los destinos. En este sentido, se plantea la necesidad que la administración pública estimula que residentes y turistas compartan la tecnología que provee el destino, lo que aportaría más información para mejorar la toma de decisiones.

Por último, consideran que no existe un SIT público, más allá de iniciativas locales puntuales. Sin embargo, plantean que se están empezando a sentar algunas bases para ello y coinciden en que la construcción de uno debe sustentarse en la colaboración públicoprivada mediante un sistema de retroalimentación que permita ir definiendo y evaluando el modelo y dimensionándolo de manera escalar. Asimismo, opinan que la sociedad está cada vez está más preparada y sensibilizada para iniciar el proceso. Sin embargo, aún quedan actores que viven en una dinámica de competición en lugar de colaboración. Por ello, todavía deben darse pasos para eliminar la desconfianza y asumir que la colabora- 
ción es más fructífera. En este sentido, apuntan la conveniencia de generar un sistema de información que incluya una selección de un número concreto de variables que respondan a los intereses colectivos en detrimento de cuestiones particulares y propias.

\section{4- CONCLUSIONES}

Las entrevistas realizadas a expertos de carácter multidisciplinar y multisectorial relacionados, estrechamente, con la generación o uso de estadística pública turística, procedentes del sector privado/público y ámbito técnico/universitario, cumplieron el objetivo de conocer la opinión relativa a la adecuación real y validez de las fuentes estadísticas, así como el papel de desempeñan en el nuevo marco territorial de los DTI. Así, el análisis de los resultados ha permitido obtener una serie de conclusiones que han sido planteadas a modo de reflexión.

En primer lugar, la opinión de los expertos revela que las relaciones de complementariedad entre la administración pública y la empresa privada en la generación de datos turísticos son totalmente necesarias. No obstante, las administraciones públicas y las empresas privadas no han logrado coordinarse y actuar de modo alineado en la generación de una metodología común de recogida de datos que permitan la toma de decisiones en planificación turística de manera inteligente. En este sentido, se propone una estrategia de creación de datos público-privada trazada mediante relaciones de colaboración entre ambas, intentando la conciliación de las distintas velocidades que cada una de ellas lleva en la generación de los datos. En esta línea se proponen los establecimientos hoteleros como pieza clave en el comportamiento del conocimiento del turista y la necesidad de recoger sus datos "inconscientes" a partir de métodos tecnológicos, reduciendo de este modo, la carga de cuestionarios a los informantes. Asimismo, se propone la conveniencia de acuerdos entre administraciones públicas y empresas locales para la recogida de datos que permitan repercusiones positivas en la economía local.

Por tanto, consideramos que, los alojamientos hoteleros, hoy en día, se convierten en uno de los yacimientos de datos más valiosos para el sector turístico, pues permiten conocer de manera precisa el comportamiento del turista. En acuerdo con los mismos, sería posible desarrollar una eficaz y provechosa fuente de datos pública. Así, la generación de datos públicos turísticos se debe realizar, en mayor medida, en dependencia de los establecimientos hoteleros al considerarlos como los actores privados que mayor capacidad reúnen para conocer al cliente, permitiendo obtener valiosa información relativa al mismo.

En segundo lugar, la administración pública debería estimular el hecho que turistas y ciudadanos compartieran datos con ella, del mismo modo que los comparten con la empresa privada. Estos permitirían la generación de una fuente de datos pública que podría ponerse a disposición de todos los actores mediante Open Data, constituyendo una nueva forma de generación de datos estadísticos que permitiría paliar el problema de recogida de datos a escala subregional. Así, sin eliminar las estadísticas turísticas públicas tradicionales, lo anterior complementaría los vacíos de información pública que ahora existen en el comportamiento del turista a partir de medios tecnológicos.

En tercer lugar, se observa el hecho que, progresivamente, los destinos van precisando de una mayor información de tipo cualitativo relativa a la motivación, experiencias y movilidad en el destino de los turistas, así como de información de la reputación del 
movilidad en el destino de los turistas, así como de información de la reputación del destino, de sus infraestructuras y de sus servicios, que complemente la información cuantitativa procedente de las fuentes estadísticas públicas. El efecto inmediato es la pérdida del papel hegemónico de los institutos estadísticos en la aportación de datos y, de información, al sistema público de información turística. Como consecuencia, deberían crearse nuevos institutos nacionales públicos de control y gestión de análisis de redes sociales que aportaran información relevante sobre cualquier línea de investigación, suponiendo una fuente de información, de tipo cualitativo, pública y complementaria a los institutos de estadística o sondeos del CIS.

Asimismo, la administración pública debe hacer un considerable esfuerzo no solo por abrir los datos turísticos generados en sistemas públicos de Open data, sino también en generar y democratizar la inteligencia de datos, poniéndola a disposición de todos los actores implicados en la planificación y gestión territorial y sectorial de los destinos turísticos. Este hecho permitiría ofrecer a la sociedad general el conocimiento necesario para la toma de decisiones, beneficiando principalmente a aquellos actores que disponen de menos recursos económicos y culturales.

Todo ello conlleva que, como planteamos inicialmente, los actuales procesos de toma de decisiones en turismo, precisen la colaboración del sector público y el privado, siendo imprescindible para su desarrollo la utilización de recursos públicos, cuya gestión es competencia de los distintos niveles de gobierno.

Sin embargo, esta manera de entender y actuar en los destinos turísticos, a través de la importancia de los datos y de la tecnología, provoca una pérdida de poder de la gestión pública local, en cuanto aparecen otros actores que desempeñan la función de su generación. Asimismo, se plantea una dualidad de instituciones locales en función de su capacidad (institucional, económica, técnica, etc.) para implementar los SIT. Del mismo modo, contribuye a que cualquier avance en el contexto de los DTI deje de ser una iniciativa política, en cuanto otros actores que cumplen la función de generación de información pueden plantearlo de manera independiente a la administración pública.

En cuarto lugar, y en relación a lo anterior, la creciente importancia de los datos turísticos y, sobre todo, las fuentes de generación de los mismos, está haciendo que los destinos turísticos necesiten replantearse las estructuras de gestión del territorio. Esto pone en riesgo la pervivencia de las tradicionales Organizaciones de Gestión de Destino (DMO) como entidades de articulación público-privada definidas por la World Travel Organization (2004) como aquellas "responsables de la gestión y/o el marketing de un destino turístico" y que no dejan de ser, en función del territorio, las conocidas como oficinas de turismo, departamentos de turismo, patronatos, consorcios, organizaciones público - privadas, con o sin ánimo de lucro, o incluso empresas privadas. Estas organizaciones han sido estructuradas tradicionalmente desde el ámbito público y orientadas principalmente a la promoción global del destino, pero cada vez se están enfocando más hacia entidades mixtas con aportación público-privada pensadas para integrar a la mayoría, generar sinergias y fomentar la competencia. Desde este punto de vista, los DMO deben utilizar la tecnología y, la información, no para vender más, sino para mejorar la experiencia del turista en cada interacción con el destino antes, durante y después del viaje. Si, actualmente, los DTI basan su diferenciación competitiva en conocer las necesidades del visitante y residente antes 
y con mayor precisión que su competencia, debemos pasar de los Destination Marketing a los Destination Management, para lo cual los datos constituyen elementos esenciales.

En definitiva, el análisis de los resultados de las entrevistas a los expertos arroja conclusiones que confirman la hipótesis planteada al comienzo de la investigación: existe un gran volumen de datos respecto al comportamiento de los turistas, no obstante, éste, en primer lugar, resulta insuficiente para la toma de decisiones inteligentes en los procesos de planificación estratégica y gestión de destinos turísticos y, en segundo lugar, se confirma que el enorme volumen de datos generados no se aprovecha para convertirlo en conocimiento. Este hecho impone la necesidad de la inmediatez de nuevos planteamientos y métodos en la recogida, uso y explotación de datos, relativa al comportamiento del turista.

Declaración responsable: La autora declara que no existe ningún conflicto de interés relacionado con la publicación de este artículo.

\section{BIBLIOGRAFÍA}

ÁLVAREZ-GAYOU J.L. (2003): Como hacer investigación cualitativa: fundamentos y metodología. México, Paidós Ibérica.

ANBALAGAN, K. y LOVELOCK, B. (2014): «The potencial for coffee tourism development in Rwanda-Neither black nor white», Tourism and hospitality Research, vol.14 (1-2), pp.81-96.

BERG, B.L. y LUNE, H. (2014): Qualitative Research Methods for the Social Sciencies ( ${ }^{\text {a }}$ edición). Harlow, Pearson Education Limited.

BLACKWELL, R., MINIARD, P.W. y ENGEL, J.F. (2002): Comportamiento del consumidor. México, Thomson.

ESCOBAR, A. y GONZÁLEZ, Y. (2011): Marketing turístico. Madrid, Síntesis.

GRAY J. y SZALAY E. (2007): eScience - a transformed scientific method. Presentation made to the NRC-CSTB. Disponible en: http://research.microsoft.com/en-us/um/ people/gray/talks/NRC-CSTB_eScience.ppt.

GRETZEL, U., YUAN, Y. y FESENMAIER, R. (2000): «Preparing for the New Economy: Advertising Strategies and Change in Destination Marketing Organizations», Journal of Travel Research, vol. 39 (2), pp.146-156.

GUTIÉRREZ, J., GARCÍA-PALOMARES, J.C., ROMANILLOS, G. y SALAS-OLMEDO, M. H. (2017): «The eruption of Airbnb in tourist cities: Comparing spatial patterns of hotels and peer-to-peer accommodation in Barcelona», Tourism Management, $\mathrm{n}^{\circ} 62$, pp. 278-291.

HEY T. ET AL. (2009): The Fourth Paradigm: Data-Intensive Scientific Discovery. Redmond, WA, Microsoft Research.

HERNÁNDEZ CARRERA, R. (2014): «La investigación cualitativa a través de entrevistas: su análisis mediante la teoría fundamentada», Cuestiones Pedagógicas, $\mathrm{n}^{\circ} 23$, pp. 187-210.

HUETE NIEVES, R., MANTECÓN TERÁN, A. y MAZÓN MARTÍNEZ, T. (2008): « ¿De qué hablamos cuando hablamos de turismo residencial? », Cuadernos de Turismo, $\mathrm{n}^{\circ} 22$, pp. 101-121. 
IVARS-BAIDAL, J.A., SOLSONA MONZONÍS, F.J. y GINER SÁNCHEZ, D. (2016): «Gestión turística y tecnologías de la información y la comunicación (TIC): El nuevo enfoque de los destinos inteligentes», Documents d'Anàlisi Geogràfica, vol. 62 (2), pp. 327-346.

KATAYAMA OMURA, R. (2014): Introducción a la investigación cualitativa: Fundamentos, métodos, estrategias y técnicas. Perú, Universidad Inca Garcilaso de la Vega.

LÓPEZ BONILLA, J.M. y LÓPEZ BONILLA, L.M. (2015): Manual de investigación de mercados turísticos. Madrid, Pirámide.

LÓPEZ PALOMEQUE, F. (2015). «Turismo y TIC. Notas sobre los procesos recientes de tecnificación de los destinos turísticos», en Libro Jubilar en Homenaje al Profesor Antonio Gil Olcina. Alicante, Universidad de Alicante, pp. 563-583.

LUQUE GIL, A., ZAYAS FERNANDEZ, B. y CARO HERRERO, J. (2015): «Los Destinos turísticos inteligentes en el marco de la inteligencia territorial: conflictos y oportunidades», Investigaciones Turísticas, $\mathrm{n}^{\circ}$ 10, pp. 1-25.

MAYER-SCHÖNBERGER, V. y CUKIER, K. (2013). Big data: A revolution that will transform how we live, work, and think. New York, Houghton Mifflin Harcourt.

MERCADO ALONSO, I. y FERNÁNDEZ TABALES, A. (2016): «La percepción del paisaje urbano como indicador de sostenibilidad turística. El caso de Sevilla», en Blàzquez, M., Mir-Gual, M., Murray, I. y Pons, G.X. (Eds.). Turismo y crisis, turismo colaborativo y ecoturismo. XV Coloquio de Geografía del Turismo, el Ocio y la Recreación de la AGE. Mon. Soc. Hist. Nat. Balears, nº 23, pp.131-142.

MILES, M.B. y HUBERMAN, A.M. (1994): Qualitative data analysis: An expanded sourcebook(2a edition). Thousand Oaks, CA, Sage.

MOROZOV, E. El País: https://elpais.com/elpais/2015/12/17/eps/1450358550_362012. html [Consulta: 16 marzo 2017].

PEÑARRUBIA ZARAGOZA, M.P. (2018): Los datos estadísticos públicos y su uso en el conocimiento del comportamiento de los turistas en Destinos Inteligentes. Tesis Doctoral dirigida por el Dr. Juan Romero y el Dr. Moisés Simancas, Valencia, Universidad de Valencia, Repositori de Contingut Lliure (RODERIC). <http://roderic.uv.es/ handle/10550/67526>

PONTING, J. y O’BRIEN, D. (2014). «Regulating "Nirvana": Sustainable surf tourism in a climate of increasing regulation», Sport Management Review, vol. 18 (1), pp. 99-110.

RITTICHAINUWAT, B. y RATTANAPHINANCHAI, S. (2015): «Applying a mixed method of quantitative and qualitative design in explaining the travel motivation of film tourists in visiting a film-shooting destination», Tourism Management, vol. 46, pp. 136-147.

RUBIN, H.J. y RUBIN, I.S. (1995): Qualitative interviewing. The art of hearing data. Thousand Oaks, CA, Sage.

SANTANA TURÉGANO, M. (2005). «Turismo, empleo y desarrollo», Papers. Revista de Sociología, $\mathrm{n}^{\circ} 77, \mathrm{pp} .79-104$.

VALLÉS M. (2009): Entrevistas cualitativas. Madrid, Centro de Investigaciones Sociológicas. 